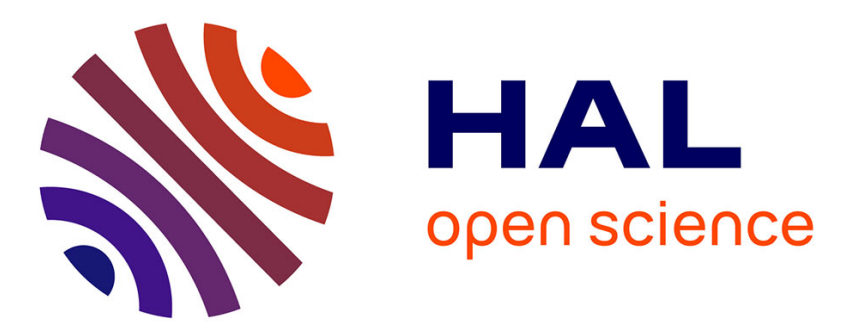

\title{
Influence de l'ordre à grande distance sur les constantes élastiques d'alliages ordonnés: application au laiton $\beta$
}

\author{
R. Conte, R. Pick
}

\section{To cite this version:}

R. Conte, R. Pick. Influence de l'ordre à grande distance sur les constantes élastiques d'alliages ordonnés: application au laiton $\beta$. Journal de Physique, 1969, 30 (5-6), pp.451-460. 10.1051/jphys:01969003005-6045100 . jpa-00206805

\section{HAL Id: jpa-00206805 https://hal.science/jpa-00206805}

Submitted on 1 Jan 1969

HAL is a multi-disciplinary open access archive for the deposit and dissemination of scientific research documents, whether they are published or not. The documents may come from teaching and research institutions in France or abroad, or from public or private research centers.
L'archive ouverte pluridisciplinaire HAL, est destinée au dépôt et à la diffusion de documents scientifiques de niveau recherche, publiés ou non, émanant des établissements d'enseignement et de recherche français ou étrangers, des laboratoires publics ou privés. 


\title{
INFLUENGE DE L'ORDRE A GRANDE DISTANGE SUR LES CONSTANTES ÉLASTIQUES D'ALLIAGES ORDONNÉS : APPLIGATION AU LAITON $\beta$
}

\author{
Par R. CONTE et R. PICK, \\ Service de Physique du Solide et de Résonance Magnétique, \\ Gentre d'Études Nucléaires de Saclay, B.P. $n^{0}$ 2, 91-Gif-sur-Yvette, France.
}

(Reçu le 4 décembre 1968.)

\begin{abstract}
Résumé. - On présente ici une étude cohérente de l'influence de l'ordre à grande distance sur le paramètre cristallin et le spectre de phonons du laiton $\beta$, en utilisant l'approximation du champ moléculaire. L'introduction de pseudo-potentiels proportionnels à la charge des ions permet alors d'expliquer la variation des coefficients élastiques de cet alliage en fonction de la température. L'accord entre la théorie et les résultats expérimentaux est satisfaisant eu égard aux approximations utilisées.
\end{abstract}

\begin{abstract}
We study the influence of the long-range order parameter on the lattice parameter and the phonon spectrum of $\beta$ brass using a molecular field approximation. By describing the ion-ion pair interaction potential through pseudo-potentials proportional to the charge of the ions, we give a complete description of the effect of temperature on the variation of the elastic constants of this material. The agreement between experiment and this calculation can be considered as satisfactory, in view of the above mentionned approximations.
\end{abstract}

I. Introduction. - Dans les alliages ordonnés dont l'ordre varie en fonction de la température, les propriétés physiques sont influencées, de façon parfois notable, par la variation de cet ordre. Parmi celles-ci, ce sont surtout la résistivité [1-5] et le pouvoir thermoélectrique [6-7] qui ont été étudiés de façon théorique, alors que de nombreuses autres données expérimentales existent, principalement pour les alliages $\mathrm{AuCu}$ et $\mathrm{AuCu}_{3}$ d'une part, CuZn d'autre part.

Nous nous sommes proposés d'étudier l'effet de l'ordre sur le spectre de phonons du laiton $\beta$. Ceci nous a amenés à nous intéresser aussi à la variation du paramètre cristallin en fonction de la température et à montrer comment la variation de la fréquence des phonons avec l'ordre modifiait les équations habituelles du champ moléculaire. Cette étude nous a permis de dégager les deux causes principales de variation des coefficients élastiques avec la température, et nous nous sommes efforcés de vérifier numériquement les résultats obtenus. Pour mener à bien ce travail, nous avons utilisé un certain nombre d'approximations, que nous énumérons ci-dessous :

1) Nous sommes restés dans le cadre de la théorie du champ moléculaire pour décrire les phénomènes d'ordre. Ceci implique que nous avons utilisé la notion d'interaction de paires entre ions, telle qu'elle a été introduite par Bragg et Williams [8].

2) Nous avons négligé l'influence de l'ordre à courte distance; ceci empêche d'utiliser nos résultats au-dessus de la température critique et immédiatement en dessous de celle-ci.

3) Pour confronter les divers résultats à notre disposition, nous avons justifié la notion d'interaction de paires par un calcul de perturbation au deuxième ordre utilisant le concept de pseudo-potentiel créé par un ion. Le manque d'information sur ces pseudopotentiels, surtout dans le cas du cuivre, nous a conduits à admettre qu'ils étaient proportionnels à la charge des ions des deux constituants.

Nous commencerons par rappeler le principe de la théorie du champ moléculaire et ses résultats habituels. Nous utiliserons ensuite celui-là pour en déduire la matrice dynamique et le tenseur d'élasticité du cristal. A l'aide d'une expression de l'énergie libre faisant intervenir les phénomènes d'ordre et les phonons, nous obtiendrons des équations décrivant le comportement du paramètre cristallin $a$ et du paramètre d'ordre $s$ avec la température. Celles-ci tiendront compte de la corrélation existant entre l'ordre et le spectre de phonons. Dans la dernière partie, nous appliquerons enfin l'hypothèse des pseudo-potentiels et comparerons la variation expérimentale des coefficients élastiques à celle que l'on peut prévoir à partir de cette hypothèse. Dans ce dernier calcul, les mesures utilisées portent d'une part sur le spectre du laiton $\beta$ à la température ordinaire, d'autre part sur la variation de son paramètre d'ordre et de son paramètre cristallin avec la température. Ce calcul montrera qu'un accord quantitatif entre les différentes mesures est encore difficile à réaliser. 
II. Théorie phénoménologique. - Considérons un alliage binaire $\mathrm{AB}$ cristallisant dans le système cubique centré et soient $f_{\mathrm{A}}$ et $f_{\mathrm{B}}$ les proportions d'atomes de types $\mathrm{A}$ et $\mathrm{B}: f_{\mathrm{A}}+f_{\mathrm{B}}=1.2 N$ sera le nombre total d'atomes du système.

Définissons le paramètre d'ordre en termes de probabilités : soient $c$ une certaine configuration de l'alliage et $\pi(\mathbf{R}, c)$ une quantité qui vaut 1 si le nœud $\mathbf{R}$ est occupé par un atome $\mathrm{A}$ et 0 dans le cas contraire. Si l'alliage présente un changement de phase ordredésordre à une température critique $T_{c}$, pour $T$ inférieur à $T_{\mathrm{c}}$ la valeur moyenne thermodynamique de $\pi(\mathbf{R}, c)$ est différente de $f_{\mathrm{A}}$. Il est alors commode de définir $\sigma(\mathbf{R}, c)$ par :

$$
\pi(\mathbf{R}, c)=f_{\mathrm{A}}[1+\sigma(\mathbf{R}, c)]
$$

et $s(\mathbf{R})$ comme sa valeur moyenne thermodynamique : $s(\mathbf{R})=\langle\sigma(\mathbf{R}, c)\rangle_{T}$.

La proportion globale d'atomes $\mathrm{A}$ étant $f_{\mathrm{A}}$, on a pour toute configuration :

$$
\sum_{\mathbf{R}}\langle\sigma(\mathbf{R}, c)\rangle_{T}=\sum_{\mathbf{R}} s(\mathbf{R})=0 .
$$

Dans le cas qui nous intéresse, le cristal s'ordonne en deux sous-réseaux cubiques simples $\mathscr{R}_{\mathrm{A}}$ et $\mathscr{R}_{\mathrm{B}}$, $\mathscr{R}_{\mathrm{A}}$ (resp. $\mathscr{R}_{\mathrm{B}}$ ) étant occupé préférentiellement par les atomes de type A (resp. B). La relation (II.1) entraîne que $s(\mathbf{R})$ vaut $s$ sur $\mathscr{R}_{\mathrm{A}}$ et $-s$ sur $\mathscr{R}_{\mathrm{B}} ; s$ est ce que nous appellerons le paramètre d'ordre. Sa variation en fonction de la température est à l'origine des effets que nous allons étudier.

II.1. RAPPEL DES PROPRIÉTÉS THERMODYNAMIQUES. - II.1.1. Énergie de configuration. - Nous supposerons que la partie $E^{\mathrm{c}}$ de l'énergie du système qui dépend de la répartition des atomes dans le cristal est une simple somme d'interactions de paires et que de plus celles-ci ne dépendent que de la distance relative des atomes et de leur type. Dans une configuration donnée, l'énergie $E^{\mathrm{c}}$ s'écrit :

$$
\begin{aligned}
& E^{\mathbf{c}}=\frac{1}{2} \sum_{\mathbf{R}, \mathbf{R}^{\prime}}\left\{j_{\mathrm{AA}}\left(\mathbf{R}-\mathbf{R}^{\prime}\right) \pi(\mathbf{R}, c) \pi\left(\mathbf{R}^{\prime}, c\right)\right. \\
& +j_{\mathrm{AB}}\left(\mathbf{R}-\mathbf{R}^{\prime}\right) \pi(\mathbf{R}, c)\left[1-\pi\left(\mathbf{R}^{\prime}, c\right)\right] \\
& +j_{\mathrm{AB}}\left(\mathbf{R}-\mathbf{R}^{\prime}\right)[1-\pi(\mathbf{R}, c)] \pi\left(\mathbf{R}^{\prime}, c\right) \\
& \left.+j_{\mathrm{BB}}\left(\mathbf{R}-\mathbf{R}^{\prime}\right)[1-\pi(\mathbf{R}, c)]\left[1-\pi\left(\mathbf{R}^{\prime}, c\right)\right]\right\} \quad(\mathrm{II} .2)
\end{aligned}
$$

où, par définition, les fonctions $j_{\mathrm{AA}}, j_{\mathrm{AB}}$ et $j_{\mathrm{BB}}$ ne dépendent que du paramètre cristallin $a$. On peut récrire (II.2) sous la forme :

$$
\begin{aligned}
E^{\mathrm{c}}=\sum_{\mathbf{R}, \mathbf{R}^{\prime}}\left\{\begin{array}{l}
D\left(\mathbf{R}-\mathbf{R}^{\prime}\right) \\
\quad+\frac{1}{2} f_{\mathrm{A}}\left[\sigma(\mathbf{R}, c)+\sigma\left(\mathbf{R}^{\prime}, c\right)\right] C\left(\mathbf{R}-\mathbf{R}^{\prime}\right)
\end{array}\right. \\
\left.+f_{\mathrm{A}}^{2} \sigma(\mathbf{R}, c) \sigma\left(\mathbf{R}^{\prime}, c\right) J\left(\mathbf{R}-\mathbf{R}^{\prime}\right)\right\} \quad(\mathrm{II} .3)
\end{aligned}
$$

avec :

$$
\begin{aligned}
2 D & =f_{\mathrm{A}}^{2} j_{\mathrm{AA}}+2 f_{\mathrm{A}} f_{\mathrm{B}} j_{\mathrm{AB}}+f_{\mathrm{B}}^{2} j_{\mathrm{BB}} \\
2 J & =\left(j_{\mathrm{AA}}+j_{\mathrm{BB}}-2 j_{\mathrm{AB}}\right) \\
C & =\left[f_{\mathrm{A}}\left(j_{\mathrm{AA}}-j_{\mathrm{AB}}\right)+f_{\mathrm{B}}\left(j_{\mathrm{AB}}-j_{\mathrm{BB}}\right)\right] .
\end{aligned}
$$

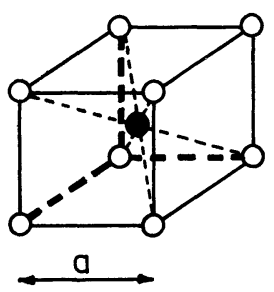

FIG. 1. - Structure ordonnée du laiton $\beta$.

Remarque : Quand les atomes sont dans leur position d'équilibre, l'invariance par translation exige que $\sum_{\mathbf{R}^{\prime}} C\left(\mathbf{R}-\mathbf{R}^{\prime}\right)$ ne dépende pas de $\mathbf{R}$; compte tenu de (II.1), le terme en $C$ s'annule alors dans (II.3). Cependant, dans la suite, nous nous intéresserons au cristal déformé par la présence de phonons; il est donc important que nous gardions ce terme.

A chaque température et pour une position donnée des ions, l'énergie totale du cristal est égale à la moyenne thermodynamique de l'expression (II.3), soit :

$$
\begin{aligned}
& E=\left\langle E^{\mathrm{c}}\right\rangle_{T}=\sum_{\mathbf{R}, \mathbf{R}^{\prime}}\left\{D\left(\mathbf{R}-\mathbf{R}^{\prime}\right)\right. \\
&+\frac{1}{2} f_{\mathrm{A}}\left[s(\mathbf{R})+s\left(\mathbf{R}^{\prime}\right)\right] C\left(\mathbf{R}-\mathbf{R}^{\prime}\right) \\
&\left.+f_{\mathbf{A}}^{2}\left\langle\sigma(\mathbf{R}, c) \sigma\left(\mathbf{R}^{\prime}, c\right)\right\rangle_{T} J\left(\mathbf{R}-\mathbf{R}^{\prime}\right)\right\} . \quad(\mathrm{II} .5)
\end{aligned}
$$

On ne sait pas calculer exactement les différentes valeurs moyennes qui interviennent dans cette dernière expression et celles-ci ne peuvent être atteintes que par l'intermédiaire d'approximations plus ou moins grossières.

Nous nous contenterons ici de rappeler les résultats habituels de celle du champ moléculaire, dont nous déduirons le spectre de phonons et le tenseur d'élasticité. La prise en compte de l'existence des phonons nous conduira enfin, toujours dans le cadre du champ moléculaire, à des expressions plus exactes des variations de $a$ et de $s$ avec la température.

II.1.2. Méthode et résultats habituels du champ moléculaire. - La valeur moyenne thermodynamique $s(\mathbf{R})$ de $\sigma(\mathbf{R}, c)$ s'obtient en minimisant l'énergie libre du réseau par rapport à $s$. Celle-ci s'écrit [5] :

$$
\begin{aligned}
& F_{\text {réseau }}(a, s, T)=2 N \mathscr{D}(a)-2 N s^{2} f_{\mathrm{A}}^{2} \mathscr{I}(a) \\
& +N k T\left[f_{\mathrm{A}}(1+s) \log (1+s)\right. \\
& +f_{\mathrm{A}}(1-s) \log (1-s)+\left(f_{\mathrm{B}}-s f_{\mathrm{A}}\right) \log \left(1-s \frac{f_{\mathrm{A}}}{f_{\mathrm{B}}}\right) \\
& \left.+\left(f_{\mathrm{B}}+s f_{\mathrm{A}}\right) \log \left(1+s \frac{f_{\mathrm{A}}}{f_{\mathrm{B}}}\right)\right]
\end{aligned}
$$


avec :

$$
\begin{aligned}
& \mathscr{D}(a)=\sum_{\mathbf{R} \in \mathscr{R}}\left[D(\mathbf{R})+D\left(\mathbf{R}+\mathbf{I}_{\mathbf{0}}\right)\right] \\
& \mathscr{I}(a)=\sum_{\mathbf{R} \in \mathscr{R}}\left[J(\mathbf{R})-J\left(\mathbf{R}+\mathbf{I}_{\mathbf{0}}\right)\right]
\end{aligned}
$$

où $\mathscr{R}$ est un sous-réseau cubique simple de côté $a$ et comprenant l'origine, et où $\mathbf{I}_{0}$ est un vecteur qui fait passer de $\mathscr{R}_{\mathrm{A}}$ à $\mathscr{R}_{\mathrm{B}}$.

Cette minimisation conduit à :

$$
s=\frac{k T}{4 f_{\mathrm{A}} \mathscr{I}(a)} \log \frac{(1+s)\left(f_{\mathrm{B}}+s f_{\mathrm{A}}\right)}{(1-s)\left(f_{\mathrm{B}}-s f_{\mathrm{A}}\right)} .
$$

La variation de $s$ est rappelée sur la figure 2 .

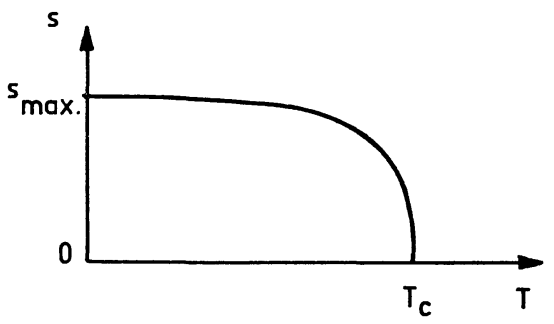

FIG. 2. $-k T_{\mathrm{c}}=2 f_{\mathrm{A}} f_{\mathrm{B}} \mathscr{I}(a) ; \quad s_{\max .}=\inf \left\{\frac{f_{\mathrm{B}}}{f_{\mathrm{A}}}, 1\right\}$.

Si on néglige les corrélations à plus de deux ions et si on décrit celles à deux ions comme une faible perturbation par rapport à l'absence de corrélation, on trouve [5] :

$$
\begin{aligned}
&\left\langle\sigma(\mathbf{R}, c) \sigma\left(\mathbf{R}^{\prime}, c\right)\right\rangle_{T} \\
&= s(\mathbf{R}) s\left(\mathbf{R}^{\prime}\right)+\delta_{\mathbf{R}, \mathbf{R}^{\prime}}[1+s(\mathbf{R})]\left[\frac{f_{\mathrm{B}}}{f_{\mathrm{A}}}-s(\mathbf{R})\right] \\
&+\left(1-\delta_{\mathbf{R}, \mathbf{R}^{\prime}}\right)\left[f_{\mathrm{B}}-f_{\mathrm{A}} s(\mathbf{R})\right][1+s(\mathbf{R})] \\
& {\left[f_{\mathrm{B}}-f_{\mathrm{A}} s\left(\mathbf{R}^{\prime}\right)\right]\left[1+s\left(\mathbf{R}^{\prime}\right)\right] \frac{J\left(\mathbf{R}-\mathbf{R}^{\prime}\right)}{k T} . }(\mathrm{II} .9)
\end{aligned}
$$

Dans cette formule, l'absence totale de corrélation que suppose le premier terme est corrigée pour $\mathbf{R}=\mathbf{R}^{\prime}$ par le deuxième; la même correction pour $\mathbf{R}$ différent de $\mathbf{R}^{\prime}$ conduit au dernier terme qui traduit l'influence de l'ordre à courte distance. Celui-ci ne joue de rôle notable qu'au voisinage de la température critique [5], région que nous ne pourrons pas étudier correctement comme nous le verrons en III. Nous négligerons donc complètement ce terme dans la suite.

II.2. SPEGTRE DE PHONONS ET TENSEUR D'ÉLASTIGITÉ. - Soit un phonon :

$$
\boldsymbol{\delta} \mathbf{R}_{\mathbf{0}}=\frac{a^{3}}{(2 \pi)^{4}} \int_{-\infty}^{+\infty} \mathrm{d} \omega \int \mathrm{d}^{3} \mathbf{q} \mathbf{a}^{\mathbf{R}_{0}}(\mathbf{q}, \omega) \mathrm{e}^{i \mathbf{q} \cdot \mathbf{R}_{0}-i \omega t}
$$

l'indice zéro étant relatif à la position d'équilibre de l'ion et le domaine d'intégration en q étant une maille du réseau réciproque du réseau ordonné. Puisqu'il y a ici deux atomes par maille, nous définissons ainsi seulement deux fonctions $\mathbf{a}^{\alpha}: \mathbf{a}^{\mathrm{A}}$ sur $\mathscr{R}_{\mathrm{A}}, \mathbf{a}^{\mathrm{B}}$ sur $\mathscr{R}_{\mathrm{B}}$.
Dans l'approximation harmonique où nous nous plaçons pour calculer ce spectre, nous négligeons les interactions entre phonons; celles-ci étant responsables des changements de configuration des ions dans le cristal, il est raisonnable d'écrire :

$$
\sigma\left(\mathbf{R}_{\mathbf{0}}+\boldsymbol{\delta} \mathbf{R}_{\mathbf{0}}, c\right)=\sigma\left(\mathbf{R}_{\mathbf{0}}, c\right) .
$$

L'accroissement d'énergie $\Delta E$ dû aux phonons est une forme quadratique des $\boldsymbol{\delta} \mathbf{R}_{\mathbf{0}}$ :

$$
\begin{aligned}
& \Delta E=\frac{1}{2} \sum_{\mathbf{R}_{0}, \mathbf{R}_{0}^{\prime}} \sum_{j, l=1}^{3}\left\{D_{j l}^{\prime \prime}\left(\mathbf{R}_{0}-\mathbf{R}_{0}^{\prime}\right)\right. \\
& \left.+\frac{f_{\mathrm{A}}\left[s\left(\mathbf{R}_{0}\right)+\right.}{2}+\left(\mathbf{R}_{0}^{\prime}\right)\right] C_{j l}^{\prime \prime}\left(\mathbf{R}_{0}-\mathbf{R}_{0}^{\prime}\right) \\
& \left.+f_{\mathrm{A}}^{2}\left\langle\sigma\left(\mathbf{R}_{0}, c\right) \sigma\left(\mathbf{R}_{0}^{\prime}, c\right)\right\rangle_{T} J_{j l}^{\prime \prime}\left(\mathbf{R}_{0}-\mathbf{R}_{0}^{\prime}\right)\right\} \\
& \quad\left(\boldsymbol{\delta} \mathbf{R}_{0}-\boldsymbol{\delta} \mathbf{R}_{0}^{\prime}\right)_{j}\left(\boldsymbol{\delta} \mathbf{R}_{0}-\boldsymbol{\delta} \mathbf{R}_{0}^{\prime}\right)_{l} . \quad(\mathrm{II} .11)
\end{aligned}
$$

La masse moyenne de l'atome situé en $\mathbf{R}_{\mathbf{0}}$ est :

$$
m\left(\mathbf{R}_{\mathbf{0}}\right)=M-m f_{\mathbf{A}} s\left(\mathbf{R}_{\mathbf{0}}\right)
$$

avec :

$M=M_{\mathrm{A}} f_{\mathrm{A}}+M_{\mathrm{B}} f_{\mathrm{B}} ; \quad m=M_{\mathrm{B}}-M_{\mathrm{A}}$

où $M_{\mathrm{A}}$ (resp. $\left.M_{\mathrm{B}}\right)$ est la masse de l'ion $\mathrm{A}$ (resp. B).

L'équation fondamentale de la dynamique s'écrit :

$$
M\left(\mathbf{R}_{0}\right) \frac{\mathrm{d}^{2}}{\mathrm{~d} t^{2}} \delta \mathbf{R}_{0}=-\operatorname{grad}_{\delta \mathbf{R}_{0}} \Delta E
$$

Puisque nous utilisons l'approximation du champ moléculaire pour calculer le terme de corrélation et que nous négligeons l'ordre à courte distance, (II.14) fournit par transformation de Fourier :

$$
\begin{gathered}
\forall \mathbf{q}: \sum_{l=1}^{3} \sum_{\beta=\mathrm{A}, \mathrm{B}} \Phi_{\alpha \beta}^{j l}(\mathbf{q}) a_{l}^{\beta}=m_{\alpha} \omega^{2} a_{j}^{\alpha} \\
j=1,2,3 ; \quad \alpha=\mathrm{A}, \mathrm{B}
\end{gathered}
$$

où :

$m_{\mathrm{A}}=M-m f_{\mathrm{A}} s ; \quad m_{\mathrm{B}}=M+m f_{\mathrm{A}} s$.

$\Phi(\mathbf{q})$ est pour tout $\mathbf{q}$ un opérateur hermitique dont chaque bloc $3 \times 3 \bar{\Phi}_{\alpha \beta}(\mathbf{q})$ est un tenseur symétrique du second ordre, à éléments réels, défini à partir des tenseurs :

$$
\begin{array}{r}
\overline{\overline{\mathscr{C}}}(\mathbf{q})=\sum_{\mathbf{k}}(\mathbf{q}+\mathbf{K}) \otimes(\mathbf{q}+\mathbf{K}) \int T(\mathbf{r}) \mathrm{e}^{-i(\mathbf{q}+\mathbf{k}) \cdot \mathbf{r}} \mathrm{d}^{3} \mathbf{r} \\
(T=D, C, J, \quad \mathscr{C}=\mathscr{D}, \mathscr{C}, \mathscr{I})
\end{array}
$$

la sommation étant étendue à tous les vecteurs du réseau réciproque du réseau désordonné. L'expression exacte des tenseurs $\bar{\Phi}(\mathbf{q})$ en fonction des tenseurs $\overline{\overline{\mathscr{C}}}(\mathbf{q})$ est sans intérêt car ce ne sont pas eux que nous utiliserons par la suite.

De (II.15), on déduit le spectre de phonons :

$\forall \mathbf{q}:$ dét $\left[\Phi_{\alpha \beta}^{j l}(\mathbf{q})-m_{\alpha} \omega^{2} \delta_{\alpha \beta} \delta^{j l}\right]=0$.

Les équations (II.15) et (II.17) se prêtent mal à l'interprétation physique; un changement de base destiné à ne garder de termes en $\omega^{2}$ que dans la diago- 
nale principale et à mettre en évidence le fait que $s$ n'est défini que par son carré met (II.17) sous la forme équivalente :

$$
\begin{gathered}
\overline{\bar{\Psi}}_{11}(\mathbf{q})=2 \overline{\overline{\mathscr{D}}}(\mathbf{q})-2 \overline{\overline{\mathscr{D}}}(\mathbf{o})+2 f_{\mathrm{A}}^{2} s^{2}\left[\overline{\overline{\mathscr{I}}}\left(\mathbf{q}+\mathbf{k}_{0}\right)-\overline{\overline{\mathscr{I}}}\left(\mathbf{k}_{0}\right)\right] \\
\overline{\bar{\Psi}}_{12}(\mathbf{q})=f_{\mathrm{A}}\left[\overline{\overline{\mathscr{C}}}(\mathbf{q})+\overline{\overline{\mathscr{C}}}\left(\mathbf{q}+\mathbf{k}_{0}\right)-\overline{\overline{\mathscr{C}}}(\mathbf{o})-\overline{\overline{\mathscr{C}}}\left(\mathbf{k}_{0}\right)\right]+f_{\mathrm{A}} \frac{m}{M} \overline{\bar{\Psi}}_{11}(\mathbf{q}) \\
\overline{\bar{\Psi}}_{22}(\mathbf{q})=2 \overline{\overline{\mathscr{D}}}\left(\mathbf{q}+\mathbf{k}_{\mathbf{0}}\right)-2 \overline{\overline{\mathscr{D}}}(\mathbf{o})+2 f_{\mathrm{A}}^{2} s^{2}\left[\overline{\overline{\mathscr{I}}}(\mathbf{q})-\overline{\overline{\mathscr{I}}}\left(\mathbf{k}_{0}\right)\right] \\
+2 f_{\mathrm{A}}^{2} \frac{m s^{2}}{M}\left[\overline{\overline{\mathscr{C}}}(\mathbf{q})+\overline{\overline{\mathscr{C}}}\left(\mathbf{q}+\mathbf{k}_{0}\right)-\overline{\overline{\mathscr{C}}}(\mathbf{o})-\overline{\overline{\mathscr{C}}}\left(\mathbf{k}_{0}\right)\right]+f_{\mathrm{A}}^{2} \frac{m^{2} s^{2}}{M^{2}} \overline{\bar{\Psi}}_{11}(\mathbf{q})
\end{gathered}
$$

avec :$$
\forall \mathbf{q} \text { : dét }\left\|\begin{array}{cc}
\overline{\bar{\Psi}}_{11}(\mathbf{q})-M \omega^{2} \overline{\overline{\mathbf{1}}} & s \overline{\bar{\Psi}}_{12}(\mathbf{q}) \\
s \overline{\bar{\Psi}}_{12}(\mathbf{q}) & \overline{\bar{\Psi}}_{22}(\mathbf{q})-\frac{M^{2}-m^{2} f_{\mathrm{A}}^{2} s^{2}}{M} \omega^{2} \overline{\overline{\mathbf{1}}}
\end{array}\right\|=0
$$

où $\mathbf{k}_{\mathbf{0}}$ est un vecteur quelconque du réseau réciproque ordonné n'appartenant pas au réseau réciproque désordonné. L'introduction de $\mathbf{k}_{\mathbf{0}}$ permet d'écrire le paramètre d'ordre sous la forme :

$$
s\left(\mathbf{R}_{\mathbf{0}}\right)=s \mathrm{e}^{i \mathbf{k}_{0} \cdot \mathbf{R}_{0}} \text {. }
$$

L'expression (II.18) appelle quelques commentaires :

a) Si l'on désirait tenir compte de l'ordre à courte distance, il suffirait d'ajouter à chaque terme de (II .18) une expression supplémentaire représentant l'influence du dernier terme de (II.9), indépendante de $\mathscr{D}$ et de $\mathscr{C}$.

b) Au-dessus de $T_{\mathrm{c}}, s=0$. Le déterminant (II.18) se factorise en deux déterminants d'ordre 3 , mais les branches « optiques » sont en fait identiques aux branches acoustiques représentées par :

$\forall \mathbf{q}:$ dét $\left[2 \overline{\overline{\mathscr{D}}}(\mathbf{q})-2 \overline{\overline{\mathscr{D}}}(\mathbf{o})-M \omega^{2} \overline{\overline{\mathbf{1}}}\right]=0$

car elles en sont déduites par une translation de $\mathbf{k}_{\mathbf{0}}$.

c) Au-dessous de $T_{\mathrm{c}}(s \neq 0)$ :

- $s$ n'intervient que par son carré. Ceci est conforme à la formule (II.8) qui est paire en $s$;

- pour un vecteur q donné, ce sont la différence des masses et le terme en $\mathscr{C}$ qui couplent les branches acoustiques et optiques : c'est ce que montre l'expression de $\overline{\bar{\Psi}}_{12}$.

d) Les fonctions $\mathscr{D}, \mathscr{C}, \mathscr{I}$ sont paires; comme elles ont la symétrie du réseau et que $2 \mathbf{k}_{0}$ est un vecteur du réseau réciproque (ce qui traduit le fait que chaque nœud est un centre de symétrie géométrique), les trois fonctions déduites de $\mathscr{D} ; \mathscr{C}$ et $\mathscr{I}$ par translation de $\mathbf{k}_{0}$ sont aussi paires.

Ainsi, lorsque $\mathbf{q}$ tend vers zéro, les tenseurs $\overline{\bar{\Psi}}_{11}(\mathbf{q})$, $\overline{\bar{\Psi}}_{12}(\mathbf{q})$ et $\bar{\Psi}_{22}(\mathbf{q})-\bar{\Psi}_{22}(\mathbf{o})$ n'ont pas de termes linéaires en $\mathbf{q}, \bar{\Psi}_{22}(\mathbf{o})$ n'est pas nul. Au voisinage de $\mathbf{q}=\mathbf{o}$, le déterminant (II.18) se factorise au quatrième ordre près en-deux déterminants d'ordre 3 .
Le premier représente les vibrations acoustiques et s'écrit :

$\mathbf{q} \rightarrow \mathbf{o}$, dét $\left[\overline{\bar{\Psi}}_{11}(\mathbf{q})-M \omega^{2} \overline{\overline{\mathbf{1}}}+\mathcal{O}\left(q^{4}\right)\right]=0$

équation où ne figurent ni $C$ ni $m$; ceci est normal car, dans la limite acoustique, le cristal doit être traité de façon macroscopique : l'invariance par translation est conservée (pas de terme en $\mathscr{C}$ ) et seule intervient la masse moyenne $M$.

(II.22) n'est rien d'autre que le déterminant classique de l'élasticité :

$$
\begin{aligned}
& \mathbf{q} \rightarrow \mathbf{o}: \text { dét }\left[\sum_{j, k=1}^{3} \lambda_{i j k l} q_{j} q_{k}\right. \\
& \left.-\frac{2 M}{a^{3}} \omega^{2} \delta_{i l}+\mathcal{O}\left(q^{4}\right)\right]=0
\end{aligned}
$$

où les $\lambda_{i j k l}$ sont les coefficients élastiques de Voigt.

L'identification au quatrième ordre près entre les deux tenseurs $\frac{2}{a^{3}} \Psi_{11}^{i l}(\mathbf{q})$ et $\sum_{j, k=1}^{3} \lambda_{i j k l} q_{j} q_{k}$ impose en général des restrictions sur les forces interatomiques. Ce n'est pas le cas ici, où nous avons affaire à des forces centrales dans un cristal cubique dont chaque atome est centre de symétrie; chaque atome est alors automatiquement en équilibre sous l'action de ces seules forces et d'une éventuelle contrainte isotrope qui ne modifie pas les équations classiques de l'élasticité.

Quant au deuxième déterminant, il décrit les vibrations optiques :

$\mathbf{q} \rightarrow \mathbf{0}:$

dét $\left[\overline{\bar{\Psi}}_{22}(\mathbf{q})-\frac{M^{2}-m^{2} f_{\mathrm{A}}^{2} s^{2}}{M} \omega^{2} \overline{\overline{\mathbf{1}}}+\mathcal{O}\left(q^{4}\right)\right]=0$.

Nous utiliserons son expression dans la troisième partie.

II.3. VARIATION DU PARAmÈtre GRISTALlin ET DU PARAMÈTRE D'ORDRE AVEG LA TEMPÉRATURE. - Dans tout ce qui précède, $a$ et $s$ doivent être considérés comme de simples paramètres qu'il nous faut maintenant déterminer simultanément. Comme nous allons 
le voir, l'ordre à grande distance influe sur la variation thermique de $a$, qui n'est pas linéaire en fonction de la température au-dessus de la température de Debye $\Theta_{\mathrm{D}}$. Les calculs du paragraphe II.2 impliquent aussi que l'énergie libre des phonons dépend de l'ordre : l'expression (II .6) est donc incomplète, et le paramètre d'ordre n'est pas donné correctement par l'équation (II.8), même dans l'approximation du champ moléculaire. Nous allons donc reprendre cette question.

Dans cette dernière approximation, l'énergie libre du cristal en présence de phonons est :

$$
F_{\text {tot. }}(a, s, T)=F_{\text {réseau }}(a, s, T)+F_{\text {phonons }}(a, s, T)
$$

$F_{\text {réseau }}$ est donné par (II.6) et dans l'approximation harmonique :

$$
F_{\text {phonons }}(a, s, T)=k T \sum \log \left|\mathrm{e}^{-\beta h \nu}-1\right|
$$

où la fréquence $\nu$ d'un phonon est fonction de $a$ et de $s$ seulement et où la sommation porte sur tous les états de phonons.

$a$ et $s$ sont solutions du système d'équations :

$$
\begin{aligned}
& \partial F_{\text {tot. }} / \partial a=0 \\
& \partial F_{\text {tot. }} / \partial s=0
\end{aligned}
$$

que nous allons résoudre de façon approchée. La première équation donne :

$\mathscr{D}^{\prime}(a)-f_{\mathrm{A}}^{2} s^{2} \mathscr{I}^{\prime}(a)+\frac{1}{2 N} \sum \frac{h \nu}{\mathrm{e}^{\beta h \nu}-1} \frac{\partial \log \nu}{\partial a}=0$.

Introduisons le coefficient $\gamma$ de Grüneisen défini par :

$$
\begin{aligned}
& \frac{\partial F_{\text {phonons }}}{\partial a}=\sum \frac{\partial \log \nu(a, s)}{\partial a} \frac{h \nu}{\mathrm{e}^{\beta h \nu}-1} \\
& \quad=\frac{\gamma(a, s, T)}{a} \sum \frac{h \nu}{\mathrm{e}^{\beta h \nu}-1}=\frac{\gamma(a, s, T)}{a} U_{\mathrm{ph} .}(a, s, T) .
\end{aligned}
$$

A $0 \circ \mathrm{K}$ où $s=f_{\mathrm{B}} / f_{\mathrm{A}}, \quad a=a_{0} \quad$ et $\quad U_{\mathrm{ph} .}=0$, (II.29) s'écrit :

$$
\mathscr{D}^{\prime}\left(a_{0}\right)=f_{\mathrm{B}}^{2} \mathscr{I}^{\prime}\left(a_{0}\right) .
$$

Dans un alliage tel que le laiton $\beta$, la température de Debye vaut environ $280^{\circ} \mathrm{K}$ [9] et est très inférieure à la température critique $T_{\mathrm{c}}=735^{\circ} \mathrm{K}$. Dans le domaine de température $\Theta_{\mathrm{D}}<T \ll T_{\mathrm{c}}$ que nous étudierons dans la troisième partie, on $\mathrm{a}$ :

$$
\frac{h \nu}{\mathrm{e}^{\beta h \nu}-1} \# k T \text {. }
$$

Pour de telles températures, $\gamma$ n'est donc plus fonction que de $a$ et $s$. Comme le paramètre cristallin varie très peu, nous pouvons nous contenter de faire un développement au premier ordre en $\left(a-a_{0}\right)$ de (II.28) qui conduit à :

$$
\begin{aligned}
& 0=\left(a-a_{0}\right)\left[\mathscr{D}^{\prime \prime}\left(a_{0}\right)-f_{\mathrm{B}}^{2} \mathscr{I}^{\prime \prime}\left(a_{0}\right)\right] \\
& +\left(f_{\mathrm{B}}^{2}-s^{2} f_{\mathrm{A}}^{2}\right) \mathscr{I}^{\prime}\left(a_{0}\right)+\frac{3}{a_{0}} \gamma\left(a_{0}, \frac{f_{\mathrm{B}}}{f_{\mathrm{A}}}\right) k T .
\end{aligned}
$$

Sur cette expression, nous voyons qu'au premier ordre l'influence de l'ordre à grande distance sur le paramètre cristallin apparaît par le seul intermédiaire du deuxième terme de (II.32).

Ce même effet avait déjà été traité de façon analogue par Dey et Sen Gupta [10]. Gependant, ces auteurs s'étaient contentés d'utiliser les deux derniers termes de (II.6) pour décrire l'énergie libre totale de leur système; l'application de l'équation (II.27 a) aurait alors dû les conduire à $\mathscr{I}^{\prime}\left(a_{0}\right)=0$. Ceci indique que, dans leur modèle, le paramètre cristallin ne varie ni avec la température, ni avec l'ordre. L'effet qu'ils obtiennent provient en fait de l'usage incorrect de certaines relations thermodynamiques.

A paramètre $a$ donné, la variation de l'ordre à grande distance en fonction de la température est décrite par l'équation (II.27 b). Celle-ci diffère de l'équation obtenue par minimisation de (II.6) par l'adjonction d'un terme supplémentaire $\partial F_{\text {phonons }} / \partial s$. Par analogie avec (II.29), nous écrirons :

$$
\begin{aligned}
& \frac{\partial F_{\text {phonons }}}{\partial s}=\sum \frac{h \nu}{\mathrm{e}^{\beta h \nu}-1} \frac{\partial \log \nu}{\partial s} \\
& \quad=s \Gamma(a, s, T) U_{\text {ph. }}(a, s, T) .
\end{aligned}
$$

Dans l'approximation du champ moléculaire, le terme $\left\langle\sigma\left(\mathbf{R}_{0}, c\right) \sigma\left(\mathbf{R}_{0}^{\prime}, c\right)\right\rangle_{T}$ de (II.11) doit être remplacé par $s\left(\mathbf{R}_{0}\right) s\left(\mathbf{R}_{0}^{\prime}\right)$; puis le spectre de phonøns calculé à partir de (II.18) est utilisé dans (II.27) pour obtenir $s(T)$. La forme que nous avons imposée au dernier membre de (II.33) résulte des expressions (II.19) sur lesquelles on voit que, dans le domaine où l'approximation (II.31) est valable, $\Gamma$ ne dépend pas de $T$ et que $\Gamma(a, o, T)$ est une quantité finie. La fonction $\Gamma(a, s, T)$ peut alors jouer le rôle d'un nouveau paramètre de Grüneisen et $s$ est solution de :

$$
\begin{array}{r}
s=\frac{f_{\mathrm{A}} k T}{4 f_{\mathrm{A}}^{2} \mathscr{I}(a)-\frac{\Gamma(a, s, T)}{N} U_{\mathrm{ph} .}(a, s, T)} \times \\
\times \log \frac{(1+s)\left(f_{\mathrm{B}}+s f_{\mathrm{A}}\right)}{(1-s)\left(f_{\mathrm{B}}-s f_{\mathrm{A}}\right)} .
\end{array}
$$

Le terme supplémentaire du dénominateur de (II .34) est analogue au terme d'échange biquadratique introduit par Rodbell [11] pour expliquer l'effet de la magnétostriction sur la variation du paramètre d'ordre dans un corps magnétique. Par l'introduction du facteur $U_{\mathrm{ph} .}(a, s, T)$, il est clair que ce terme joue surtout un rôle important au-dessus de $\Theta_{\mathrm{D}}$, et cela d'autant plus que l'on est au voisinage de $T_{\mathrm{c}}$. L'influence de ce terme ne peut donc pas être entièrement négligée. Il nous sera alors plus facile pour la suite de 
décrire la variation de $a$ en utilisant l'équation (II.32) dans laquelle $s^{2}$ prend sa valeur expérimentale.

L'ensemble des équations (II .22), (II.24) et (II.32) va nous permettre maintenant de relier entre elles diverses mesures relatives au laiton $\beta$. C'est ce que nous allons faire dans la troisième partie.

III. Application au laiton $\beta$. - III.1. IntroducTION. - L'exploitation de la théorie précédente nécessite de nombreux résultats expérimentaux obtenus sur le même alliage. A notre connaissance, seules les mesures faites sur le laiton $\beta$ sont actuellement assez complètes pour pouvoir être exploitées. Même pour cet alliage, le nombre et la qualité des expériences à notre disposition rendent impossible une détermination convenable des fonctions $D, C$ et $J$. Nous serons donc amenés d'une part à n'utiliser les mesures du spectre de phonons qu'au centre de la zone de Brillouin, d'autre part à faire une approximation très grossière permettant de relier entre elles ces différentes fonctions.

Nous commencerons par indiquer quelle approximation nous avons utilisée pour décrire l'ensemble de ces trois fonctions, et par donner l'expression des courbures optiques et acoustiques du spectre de phonons que l'on en déduit pour $\mathbf{q}=\mathbf{0}$. Nous discuterons ensuite la valeur des expériences dont nous disposons et finirons en comparant la variation expérimentale des coefficients élastiques en fonction de la température aux résultats que l'on peut attendre de la théorie du champ moléculaire.

III.2. INTERAGTIONS DE PAIRES ET PSEUDO-POTENTIEL. - Soit $c$ une configuration des atomes A et B. L'hamiltonien décrivant le système des électrons de conduction de l'alliage s'écrit, dans l'approximation à un électron du champ self-consistant de Hartree :

$$
\begin{aligned}
H^{\mathrm{c}}= & -\frac{\hbar^{2}}{2 m_{\mathrm{e}}} \Delta-e\left[\sum _ { \mathbf { R } } \left\{\pi(\mathbf{R}, c) v_{\mathrm{A}}(\mathbf{r}-\mathbf{R})\right.\right. \\
& \left.\left.+[1-\pi(\mathbf{R}, c)] v_{\mathbf{B}}(\mathbf{r}-\mathbf{R})\right\}+V_{\mathrm{s}}^{\mathrm{c}}(\mathbf{r})\right] \\
= & -\frac{\hbar^{2}}{2 m_{\mathrm{e}}} \Delta+W^{\mathrm{c}}(\mathbf{r})
\end{aligned}
$$

où $v_{\mathrm{A}}\left(\right.$ resp. $\left.v_{\mathrm{B}}\right)$ est le potentiel créé par l'ion $\mathrm{A}$ (resp. $\left.\mathrm{B}\right)$ de charge $e z_{\mathrm{A}}\left(\right.$ resp. $e z_{\mathrm{B}}$ ) et où $V_{\mathrm{s}}^{\mathrm{c}}$ est le potentiel selfconsistant d'écran dû aux électrons de conduction lorsque la configuration des ions est $c$.

Nous nous contentons ici de faire un calcul de perturbation au deuxième ordre en prenant $W^{\mathrm{c}}$ pour potentiel perturbateur; l'énergie totale du système s'écrit alors :

$$
E_{\text {totale }}^{\mathrm{c}}=E_{0}-\frac{a^{3} \varepsilon_{0}}{2} \sum_{\mathbf{q} \neq \mathbf{o}}\left[1-\frac{\varepsilon_{0}}{\varepsilon(\mathbf{q})}\right] q^{2}\left|V^{\mathrm{c}}(\mathbf{q})\right|^{2}+E_{\mathrm{W}}^{\mathrm{c}} \text {. }
$$

Dans cette expression :

- $E_{0}$ est une constante indépendante de la configuration des ions;
- $\varepsilon(\mathbf{q})$ est la constante diélectrique d'un gaz d'électrons libres :

$\varepsilon(\mathbf{q})=\varepsilon_{0}+\frac{m_{\mathrm{e}} e^{2}}{4 \pi \hbar^{2}} \frac{2 k_{\mathrm{F}}}{\pi q^{2}}\left[1+\frac{4 k_{\mathrm{F}}^{2}-q^{2}}{4 q k_{\mathrm{F}}} \log \left|\frac{q+2 k_{\mathrm{F}}}{q-2 k_{\mathrm{F}}}\right|\right]$

- $\varepsilon_{0}=[\varepsilon(\mathbf{q})]_{q=\infty}$ est la constante diélectrique du vide qui vaut $1 / 4 \pi$ unités C.G.S. :

$$
\begin{aligned}
V^{\mathrm{c}}(\mathbf{q}) & =\frac{1}{a^{3}} \sum_{\mathbf{R}} \int\left\{\pi(\mathbf{R}, c) v_{\mathrm{A}}(\mathbf{r}-\mathbf{R})\right. \\
& \left.+(1-\pi(\mathbf{R}, \mathbf{c})) v_{\mathrm{B}}(\mathbf{r}-\mathbf{R})\right\} \mathrm{e}^{-i \mathbf{q} \cdot \mathbf{r}} \mathrm{d}^{3} \mathbf{r}
\end{aligned}
$$

et $E_{\mathrm{W}}^{\mathrm{c}}$ est l'énergie électrostatique d'un ensemble électriquement neutre constitué des charges ioniques ponctuelles e $\left\{\pi(\mathbf{R}, c) z_{\mathrm{A}}+(1-\pi(\mathbf{R}, c)) z_{\mathrm{B}}\right\}$ et d'un gaz d'électrons libres.

En développant les deux derniers termes de (III.3) et en regroupant les termes indépendants de la configuration, nous obtenons une expression identique à (II.2) dans laquelle l'interaction entre des ions situés en des nœuds $\mathbf{R}$ et $\mathbf{R}^{\prime}$ s'écrit :

$$
\begin{aligned}
& j_{\mathrm{MN}}\left(\mathbf{R}-\mathbf{R}^{\prime}\right)=\frac{a^{6}}{(2 \pi)^{3}} \varepsilon_{0} \int \mathrm{e}^{i \mathbf{q} .\left(\mathbf{R}-\mathbf{R}^{\prime}\right)} \\
& \left(\left[\frac{e}{a^{3} \varepsilon_{0}}\right]^{2} \frac{z_{\mathrm{M}} z_{\mathrm{N}}}{q^{2}}-\left[1-\frac{\varepsilon_{0}}{\varepsilon(\mathbf{q})}\right] q^{2} v_{\mathrm{M}}(\mathbf{q}) v_{\mathrm{N}}(-\mathbf{q})\right) \mathrm{d}^{3} \mathbf{q}
\end{aligned}
$$

$\mathrm{M}, \mathrm{N}=\mathrm{A}, \mathrm{B}$.

Lorsque $\mathbf{q}$ tend vers zéro, $v_{\mathrm{M}}(\mathbf{q})$ se comporte comme $\frac{e}{a^{3} \varepsilon_{0}} \frac{z_{\mathrm{M}}}{q^{2}}$, car le potentiel créé par un ion est purement coulombien à grande distance de celui-ci; $j_{\text {MN }}(\mathbf{q})$ tend alors vers une constante, ce qui conduit à une interaction de paires à portée finie, aux oscillations de Friedel près.

Un tel calcul suppose que $v_{\mathrm{A}}$ et $v_{\mathrm{B}}$ peuvent être traités indépendamment comme une faible perturbation du gaz d'électrons libres. On sait que pour les métaux simples une telle approximation est valable et permet de prédire avec une assez bonne précision le spectre de phonons de ceux-ci à partir de pseudopotentiels calculés de façons indépendantes. A priori, un tel raisonnement ne s'applique pas au cuivre dont les électrons $3 d$ se recouvrent d'un ion à l'autre. Cependant, comme le cuivre et le zinc se suivent dans le tableau périodique, leurs couches internes sont peu différentes, ce qui rend sans doute moins déraisonnable l'approche de $v_{\mathrm{Cu}}$ et $v_{\mathrm{Zn}}$ par des pseudo-potentiels. Comme la notion de pseudo-potentiel permet de tenir compte de la structure des couches internes, nous pouvons nous proposer de découpler l'influence de celles-ci et de la charge de l'ion en écrivant :

$$
\frac{v_{\mathrm{Cu}}}{z_{\mathrm{Cu}}}=\frac{v_{\mathrm{Zn}}}{z_{\mathrm{Zn}}}=v
$$

où $v$ est indépendant de la charge $z$. 
Nous avons vérifié la validité d'une telle hypothèse en comparant le spectre de phonons mesuré à celui résultant d'un calcul dans lequel $v$ possède trois paramètres ajustables; ce calcul reproduit la forme générale du spectre avec une erreur de $20 \%$ sur $\omega$. Nous adopterons donc pour la suite l'hypothèse (III.7), ce qui fait prendre à l'interaction de paires (III.6) la forme très simple :

$$
j_{\mathrm{MN}}\left(\mathbf{R}^{\prime}-\mathbf{R}^{\prime}\right)=z_{\mathrm{M}} z_{\mathrm{N}} f\left(\mathbf{R}-\mathbf{R}^{\prime}\right) .
$$

III.3. ApPlication DE L'HYPOTHÈSE DES PSEUdopotentiels aU gas du laiton $\beta$. - Dans le modèle développé plus haut, le tenseur d'élasticité a pour composantes, toutes questions de symétrie mises à part :

$$
\lambda_{i j k l}=\frac{1}{a^{3}} \partial_{j} \partial_{k} \Psi_{11}^{i l}(\mathbf{o}) .
$$

Étant donné l'analogie entre (II.23) et (II.24), nous définissons de même un tenseur de courbure optique de composantes :

$$
\Lambda_{i j k l}=\frac{M}{M^{2}-m^{2} f_{\AA}^{2} s^{2}} \partial_{j} \partial_{k} \Psi_{22}^{i l}(\mathbf{o}) .
$$

Compte tenu de (III.7) et des expressions de $D, C$ et $J$ données par (II.4), on a :

$\forall \mathbf{r}: \frac{J(\mathbf{r})}{D(\mathbf{r})}=\frac{1}{\left(1+f_{\mathrm{Zn}}\right)^{2}}=\lambda, \quad \frac{C(\mathbf{r})}{D(\mathbf{r})}=\frac{-2}{1+f_{\mathrm{Zn}}}=\mu$.

Les coefficients d'élasticité et les composantes du tenseur de courbure optique se mettent alors sous la forme :

$$
\begin{aligned}
& \lambda_{i j k l} \equiv c_{\alpha \beta}=\varphi_{\alpha \beta}(a)+s^{2} \psi_{\alpha \beta}(a) \\
& \Lambda_{i j k l} \equiv K_{\alpha \beta}=\frac{a^{3}}{M\left(M^{2}-m^{2} f_{\AA}^{2} s^{2}\right)} \\
& {\left[\left(\lambda M^{2}+\mu m M+m^{2}\right) f_{\mathrm{A}}^{2} s^{2} \varphi_{\alpha \beta}(a)\right.} \\
& \left.+\frac{\left(M^{2}+\mu m M f_{\mathrm{A}}^{2} s^{2}+m^{2} f_{\mathrm{A}}^{4} s^{4}\right)}{\lambda f_{\mathrm{A}}^{2}} \psi_{\alpha \beta}(a)\right] . \quad \text { (III. 12) }
\end{aligned}
$$

Dans les directions des axes de symétrie du cristal et dans celles-là seulement, une branche optique est couplée avec une branche acoustique déterminée; dans ce cas, la courbure de la branche optique en $\mathbf{q}=\mathbf{o}$ est donnée par la même combinaison linéaire des $\Lambda_{i j k l}$ que celle qui, à partir des $\lambda_{i j k l}$, donne la vitesse du son pour la direction et la polarisation considérées.

Les formules (III.12) vont servir de base à notre comparaison entre les diverses mesures à notre disposition; nous allons maintenant rappeler ces dernières en en faisant simultanément la critique.

III.4. Expériences a NOTRE Disposition. - Le paramètre d'ordre $s$ a été mesuré en fonction de la température par diffraction de neutrons [12] : sur un échantillon où $f_{\mathrm{Cu}}=0,53$, on connaît ainsi $s$ à $3 \%$ près pour $T_{\mathrm{c}}-T$ supérieur à $50^{\circ}$.

Les mesures de la variation du paramètre cristallin [13] en fonction de $T$ remontent à 1934 : elles mettent malheureusement peu en évidence la part de variation qui est imputable à l'ordre, car il s'agit là de mesures absolues, alors que des mesures différentielles seraient souhaitables étant donné la petitesse de la dilatation anomale. Il ne nous a donc pas été possible de vérifier de façon précise la validité de l'équation (II.32). Nous obtiendrons seulement une estimation du coefficient de $\left(f_{\mathrm{B}}^{2} s^{2}-f_{\mathrm{A}}^{2}\right)$ dans cette équation, et utiliserons par ailleurs les résultats globaux de ces mesures.

Le spectre de phonons du laiton $\beta$ a été mesuré [9] à la température ordinaire $\left(T_{0}=296 \mathrm{~K}\right)$ pour une concentration $f_{\mathrm{Cu}}=0,525 \pm 0,005$; chaque fréquence est connue avec une précision de 2 ou $3 \%$. Nous n'utiliserons ces mesures que pour obtenir les courbures des fréquences optiques et acoustiques au centre de la zone de Brillouin. Gilat et Dolling ont proposé plusieurs modèles de forces qui donnent une description convenable du spectre de phonons mesuré. C'est leur modèle $4 E$, correspondant à des forces entre proches voisins, que nous utiliserons pour obtenir les valeurs numériques de $K_{\alpha \beta}$ et $c_{\alpha \beta}$, donc, par (III.12), celles $\operatorname{de} \varphi_{\alpha \beta}\left(a_{0}\right)$ et $\psi_{\alpha \beta}\left(a_{0}\right)$.

Nous nous servirons enfin des mesures [14] de la variation des coefficients élastiques en fonction de $T$, faites sur un échantillon de concentration $f_{\mathrm{Cu}}=0,50$ et qui sont reproduites sur les figures 3,4 et 5 : il se trouve qu'à cette composition la phase ordonnée $\beta^{\prime}$ se transforme en un mélange de phases $\beta^{\prime}$ et $\gamma$ avant $T_{c}$, à une température d'environ $650 \mathrm{o}$. C'est très vraisemblablement la raison pour laquelle $\frac{c_{11}-c_{12}}{2}$ possède vers $660 \mathrm{~K}$ un changement du signe de sa courbure, alors que la théorie précédente ne laisse nullement prévoir un tel changement avant le voisinage immédiat de $T_{\mathrm{c}}$. Nous ne pouvons donc attribuer aucun crédit

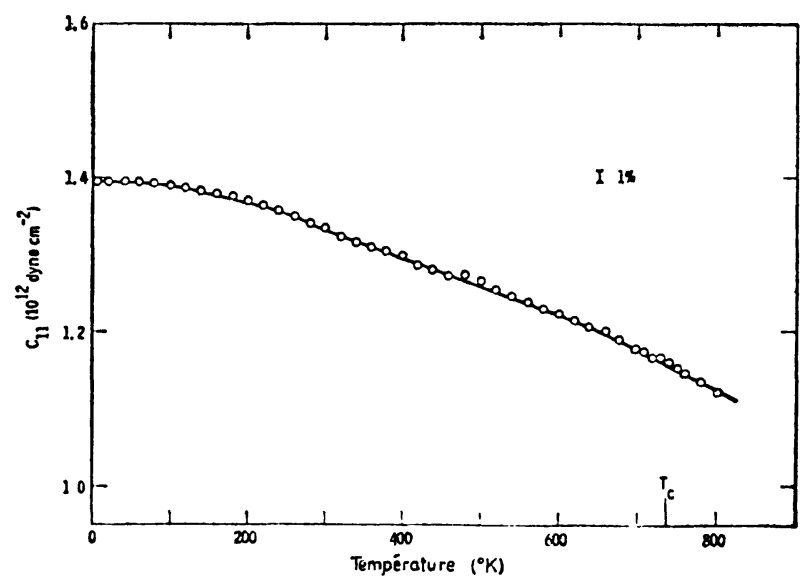

FIG. 3. - Variation de $c_{11}$ avec la température d'après McManus [14]. 


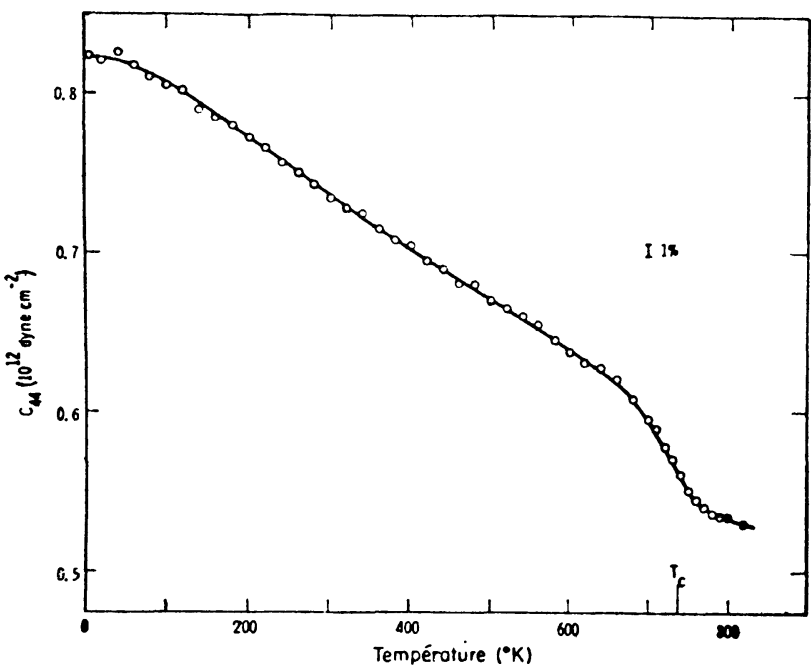

FIG. 4. - Variation de $c_{44}$ avec la température d'après McManus [14].

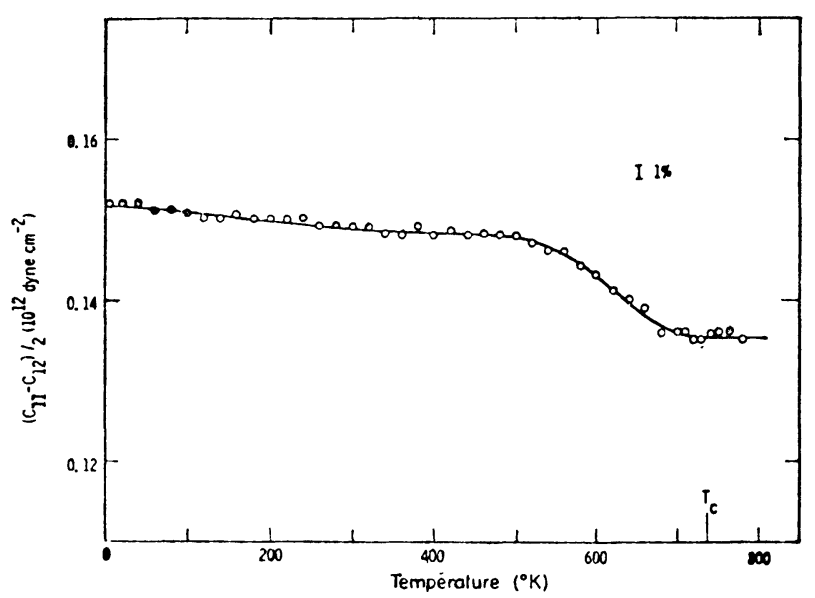

FIG. 5. - Variation de $\frac{c_{11}-c_{12}}{2}$ avec la température d'après McManus [14].

à la curieuse variation de $c_{44}(f g .4)$ avec la température au-dessus de $660 \mathrm{~K}$; seule la région où $\frac{\mathrm{d} c_{44}}{\mathrm{~d} T}$ est approximativement constant pourra être comparée à nos calculs. Notons enfin que $c_{11}$ ne semble nullement affecté par la transformation que nous admettons pour expliquer les mesures de McManus; ceci n'infirme pas notre explication car $c_{11}$ dépend surtout de la courbure du pseudo-potentiel autour de $\mathbf{q}=\mathbf{0}$ et dépend peu de la structure et de l'ordre régnant dans le cristal.

Il faut par ailleurs remarquer que, lors des mesures de McManus, le paramètre d'ordre $s$ n'a vraisemblablement pas atteint sa valeur d'équilibre à la température ordinaire par suite d'un refroidissement trop rapide des échantillons employés. La valeur réellement atteinte $s_{\max }$. peut se déterminer en cherchant en dessous de quelle température les coefficients élastiques varient linéairement avec la température. En appliquant cette technique à $\frac{c_{11}-c_{12}}{2}$, coefficient le moins sensible à la variation du paramètre cristallin, nous avons trouvé $s_{\max }=0,94$, ce qui apparaît comme une valeur très plausible : en dessous de $540 \mathrm{~K}$, l'ordre reste bloqué à cette valeur.

A l'aide de ces différentes mesures, nous allons calculer pour $T_{0}=296{ }^{\circ} \mathrm{K}$ la valeur des coefficients $\varphi_{\alpha \beta}$ et $\psi_{\alpha \beta}$. En utilisant par ailleurs les mesures de $a(T)$, de $s(T)$ ainsi que les dérivées $c_{\alpha \beta}^{\prime}\left(T_{0}\right)$, nous serons capables de déterminer les variations de $c_{\alpha \beta}$ avec la température. Ce sont ces valeurs calculées de $c_{\alpha \beta}(T)$ que nous comparerons aux mesures de McManus dans le domaine de température $296 \circ \mathrm{K}<T<640$ K.

III.5. Comparaison entre Les diverses mesures EXPÉRIMENTALES. - Les données numériques de constantes de forces [9] permettent de calculer $c_{\alpha \beta}\left(T_{0}\right)$ et $K_{\alpha \beta}\left(T_{0}\right)$, puis, par l'intermédiaire de (III.12), $\varphi_{\alpha \beta}\left(a_{0}\right)$ et $\psi_{\alpha \beta}\left(a_{0}\right)$, le paramètre d'ordre $s\left(T_{0}\right)$ étant pris égal à sa valeur maximale $f_{\mathrm{Zn}} / f_{\mathrm{Cu}}$; le tableau I consigne ces résultats en indiquant en outre les branches du spectre pour lesquelles les courbures dépendent de ce seul coefficient $\left.{ }^{(}\right)$.

\section{TABLEAU I}

$\begin{array}{cccc}(\alpha, \beta) & 1,1 & 4,4 & \frac{1,1-1,2}{2} \\ - & - & - & -\end{array}$

$\begin{array}{cccc}\text { Branche } & (\zeta, 0,0) L & (\zeta, 0,0) T & (\zeta, \zeta, 0) T_{2} \\ & & (\zeta, \zeta, 0) T_{1} & \\ K_{\alpha \beta}\left(T_{0}\right) & -3,92 & -10,48 & 14,85 \\ c_{\alpha \beta}\left(T_{0}\right) & 13,14 & 7,43 & 1,09 \\ & (13,34) & (7,38) & (1,49) \\ \psi_{\alpha \beta}\left(T_{0}\right) & -0,367 & -0,646 & 0,776\end{array}$

Valeur des constantes optiques $\left(K_{\alpha \beta}\right)$, élastiques $\left(c_{\alpha \beta}\right)$ et de $\psi_{\alpha \beta}$ déduites du modèle $4 E$ de Gilat et Dolling [9] pour décrire le spectre de phonons à $T_{0}=296 \mathrm{oK}$ et une concentration $f_{\mathrm{Cu}}=0,52$. Les valeurs entre parenthèses représentent les mesures de McManus [14] avec une concentration $t_{\mathrm{Cu}}=0,50$. Toutes les valeurs sont en unités $10^{10} \mathrm{~N} / \mathrm{m}^{2}=10^{11}$ dynes $/ \mathrm{cm}^{2}$.

Comme nous ne pouvons utiliser qu'un domaine de température où le paramètre d'ordre varie peu, il est raisonnable que nous nous contentions de développer $c_{\alpha \beta}$ au premier ordre en $\left(a-a_{0}\right)$ et $\left(s^{2}-s_{0}^{2}\right)$, l'indice 0 correspondant à la température $T_{0}$ à laquelle a été mesuré le spectre de phonons. On a alors :

$$
\begin{aligned}
c_{\alpha \beta}(T)-c_{\alpha \beta}\left(T_{0}\right)=\left(a-a_{0}\right)\left[\varphi_{\alpha \beta}^{\prime}\left(a_{0}\right)+s_{0}^{2} \psi_{\alpha \beta}^{\prime}\left(a_{0}\right)\right] \\
+\left(s^{2}-s_{0}^{2}\right) \psi_{\alpha \beta}\left(a_{0}\right)
\end{aligned}
$$

(1) $\varphi_{\alpha \beta}\left(a_{0}\right)$, qui n'est pas nécessaire pour la suite, n'a pas été reporté dans ce tableau. 
où, d'après (II.32) :

$$
a-a_{0}=\gamma\left(T-T_{0}\right)+\delta\left(s^{2}-s_{0}^{2}\right)
$$

ce qui conduit à :

$$
\begin{aligned}
c_{\alpha \beta}^{\prime}\left(T_{0}\right)=\left[\varphi_{\alpha \beta}^{\prime}\left(a_{0}\right)+s_{0}^{2} \psi_{\alpha \beta}^{\prime}\left(a_{0}\right)\right] a^{\prime}\left(T_{0}\right) \\
+2 s_{0} s_{0}^{\prime} \psi_{\alpha \beta}\left(a_{0}\right) .
\end{aligned}
$$

A la température ordinaire $T_{0}, s_{0}^{\prime}$ est très petit et peut être négligé dans (III.15). On en déduit le développement de $c_{\alpha \beta}$ :

$$
\begin{array}{r}
c_{\alpha \beta}(T)-c_{\alpha \beta}\left(T_{0}\right)=\frac{a-a_{0}}{\gamma} c_{\alpha \beta}^{\prime}\left(T_{0}\right)+\left(s^{2}-s_{0}^{2}\right) \psi_{\alpha \beta}\left(a_{0}\right) \\
=\left(T-T_{0}\right) c_{\alpha \beta}^{\prime}\left(T_{0}\right)+\left[\psi_{\alpha \beta}\left(a_{0}\right)+\frac{\delta}{\gamma} c_{\alpha \beta}^{\prime}\left(T_{0}\right)\right] \\
\times\left(s^{2}-s_{0}^{2}\right)
\end{array}
$$

expression dans laquelle nous avons mis en évidence le terme linéaire en température et celui qui dépend de l'ordre. $c_{\alpha \beta}^{\prime}\left(T_{0}\right)$ se déduit des mesures de McManus [14] et $\gamma$ et $\delta$ de celles de Owen et Pickup [13] :

$$
\gamma=6,49 \times 10^{-5} \AA / \mathrm{d}^{\mathrm{o}} ; \quad \delta=-3,43 \times 10^{-3} \AA \text {. }
$$

\section{TABLEAU II}

$$
\begin{array}{cccc}
(\alpha, \beta) & 1,1 & 4,4 & \frac{1,1-1,2}{2} \\
- & - & - & - \\
\psi_{\alpha \beta}\left(a_{\theta}\right) & -0,367 & -0,646 & +0,776 \\
\frac{\delta}{\gamma} c_{\alpha \beta}^{\prime}\left(T_{\theta}\right) & +0,209 & +0,181 & +0,0047 \\
\psi_{\alpha \beta}\left(a_{0}\right)+\frac{\delta}{\gamma} c_{\alpha \beta}^{\prime}\left(T_{0}\right) & -0,158 & -0,465 & +0,781 \\
\left(T_{1}-T_{0}\right) c_{\alpha \beta}^{\prime}\left(T_{0}\right) & -1,361 & -1,180 & -0,0306
\end{array}
$$

Valeur des différents coefficients entrant dans la formule (III. 17) en $10^{10} \mathrm{~N} / \mathrm{m}^{2}=10^{11}$ dynes $/ \mathrm{cm}^{2}$. Dans la dernière ligne, $T_{1}=640 \mathrm{o}$.

Nous avons rassemblé dans le tableau II les valeurs comparées des deux termes de (III.16) pour
$T_{1}=640{ }^{\circ} \mathrm{K}$ qui est la température maximale où les mesures de McManus [14] sont utilisables.

C'est la part des variations de $c_{\alpha \beta}$ imputable à l'ordre qui nous intéresse ici; dans notre approximation, celle-ci est représentée par le terme en $\left(s^{2}-s_{0}^{2}\right)$ de (III.16). D'après le tableau II, pour $c_{11}$ et $c_{44}$, l'effet de l'ordre est masqué par le terme linéaire en $T$ : seules des mesures très fines pourront le déceler et son effet sera de donner une courbure positive aux courbes représentatives de $c_{11}$ et $c_{44}$, tout au moins tant que l'influence de l'ordre à courte distance sera négligeable. En revanche, sur $\frac{c_{11}-c_{12}}{2}$, l'ordre a une importance relative environ 100 fois plus grande que sur $c_{11}$ et $c_{\mathbf{4 4}}$. En outre, comme l'indique le tableau II, le coefficient de $\left(s^{2}-s_{0}^{2}\right)$ ne dépend pratiquement que de $\psi_{\alpha \beta}\left(a_{0}\right)$; il est donc connu avec une bonne précision sous réserve de l'hypothèse (III.11) : ces circonstances réunies nous ont permis de tracer une courbe théorique des variations de $\frac{c_{11}-c_{12}}{2}$ dans le domaine de température $\left(T_{0}, T_{1}\right)$.

Dans le tableau III, nous avons représenté les variations de $\frac{c_{11}-c_{12}}{2}$ que nous comparons à leur valeur expérimentale, en séparant les termes d'ordre et de

\begin{tabular}{|c|c|c|c|c|c|c|c|c|}
\hline \multirow[b]{2}{*}{$T$} & \multirow[b]{2}{*}{$\left(T-T_{0}\right)_{-}{ }^{*} c^{\prime}\left(T_{0}\right)$} & $s_{0}=$ & $\begin{array}{l}\text { Conditions } \\
\text { McManus } \\
94 ; f_{\mathrm{Cu}}=0,50\end{array}$ & \multicolumn{3}{|c|}{$c(T)-c\left(T_{0}\right)$} & \multicolumn{2}{|c|}{$\begin{array}{c}\text { CONDITIONS } \\
\text { D'ÉQUILIBRE } \\
s_{0}=1 ; f_{\mathrm{Cu}}=0,50\end{array}$} \\
\hline & & $s$ & $\begin{array}{c}\left(s^{2}-s_{0}^{2}\right) \times \\
{\left[\psi\left(a_{0}\right)+\frac{\delta}{\gamma} c^{\prime}\left(T_{0}\right)\right]}\end{array}$ & $\begin{array}{l}\text { CALCULÉ } \\
\text { MCMANUS }\end{array}$ & $\begin{array}{l}\text { MESURÉ } \\
\text { MCMANUS }\end{array}$ & $\begin{array}{c}\text { CALCULÉ } \\
\text { ÉQUILIBRE }\end{array}$ & {$\left[\begin{array}{c}\left(s^{2}-s_{0}^{2}\right) \times \\
\psi\left(a_{0}\right)+\frac{\delta}{\gamma} c^{\prime}\left(T_{0}\right)\end{array}\right]$} & $s(T)$ \\
\hline - & - & - & 一 & 一 & - & - & - & - \\
\hline 296 & 0 & 0,94 & 0 & 0 & 0 & 0 & 0 & 1 \\
\hline 520 & $-0,0199$ & 0,94 & 0 & $-0,0199$ & $-0,0199$ & $-0,077$ & $-0,0572$ & 0,963 \\
\hline 550 & $-0,0225$ & 0,94 & 0 & $-0,0225$ & $-0,0225$ & $-0,107$ & $-0,0850$ & 0,945 \\
\hline 580 & $-0,0252$ & 0,905 & $-0,0505$ & $-0,0757$ & $-0,0382$ & $-0,167$ & $-0,142$ & 0,905 \\
\hline 610 & $-0,0279$ & 0,859 & $-0,1148$ & $-0,143$ & $-0,0614$ & $-0,234$ & $-0,206$ & 0,859 \\
\hline 640 & $-0,0306$ & 0,795 & $-0,1976$ & $-0,228$ & $-0,0873$ & $-0,320$ & $-0,289$ & 0,795 \\
\hline $35=1$ & & 0 & & & & & & 0 \\
\hline
\end{tabular}
température. Nous voyons que, contrairement au cas de $c_{11}$ et de $c_{44}$, d'une part le terme qui dépend de l'ordre est beaucoup plus important que celui qui n'en dépend pas, d'autre part il contribue à diminuer la valeur $\frac{c_{11}-c_{12}}{2}$ (courbure négative). Dans ce même tableau, nous avons reporté les valeurs de $\frac{c_{11}-c_{12}}{2}$ que nous calculerions pour un alliage dans lequel l'équilibre thermodynamique serait atteint même à basse température. Ces dernières valeurs sont seulement indicatives, car la comparaison entre les résultats expé-

\section{TABLEAU III}

Valeurs de $\frac{c_{11}-c_{12}}{2}$ (unités $10^{10} \mathrm{~N} / \mathrm{m}^{2}$ ) calculées et mesurées pour $f_{\mathrm{Cu}}=0,50$. 
rimentaux et calculés montre que nous obtenons un coefficient de $\left(s^{2}-s_{0}^{2}\right)$ environ trois fois plus grand que celui déduit des résultats de McManus [14] et Roussel [12].

La raison principale de ce désaccord provient certainement des éq. (III.11) et (III.12). Dans le laiton $\beta$ où la différence relative des masses entre les deux constituants est d'environ un centième, le comportement de l'équation (III.12) est dominé par le terme en $\lambda$. Nos résultats indiquent que l'hypothèse de proportionnalité entre les potentiels d'interaction $D$ et $J$ permet mal de décrire convenablement les courbures acoustiques et optiques en $\mathbf{q}=\mathbf{0}$.

IV. Conclusion et remarques. - Nous avons présenté une étude de l'influence des phénomènes d'ordre à grande distance sur le paramètre cristallin et le spectre de phonons d'alliages binaires, et nous avons cherché à relier entre elles ces différentes mesures.

L'ensemble des équations que nous avons développées dans la deuxième partie a un caractère phénoménologique. Nous avons fait intervenir les phénomènes d'ordre de deux façons distinctes dans l'étude du spectre de phonons : d'une part, directement, dans la matrice dynamique, d'autre part en étudiant leur influence sur la dilatation du réseau. Cette description est à l'opposé de celle proposée par Shmatov [15] qui a montré que la variation du paramètre d'ordre sous l'effet des contraintes introduisait un terme supplémentaire dans les coefficients élastiques. Cet effet existe lorsque le paramètre d'ordre atteint sa valeur d'équilibre en un temps suffisamment rapide pour que l'ordre « suive " la déformation créée par un phonon. Dans les mesures faites par McManus, où la fréquence des ondes utilisées pour mesurer les coefficients élastiques est environ $10^{7} \mathrm{~Hz}$, cet effet ne joue qu'au voisinage du point critique, région pour laquelle l'influence de termes tels que l'ordre à courte distance ne peut pas non plus être négligée. Le terme de Shmatov doit donc être négligé dans notre étude, comme nous l'avons explicitement fait dans la deuxième partie. Les deux termes que nous avons introduits peuvent être du même ordre de grandeur et de signes opposés, comme c'est le cas pour $c_{11}$ et $c_{44}$.

Ainsi que nous l'avons vu dans la troisième partie, il est difficile de faire à l'heure actuelle des comparaisons entre les diverses mesures à notre disposition et nous n'avons pas obtenu un bon accord quantitatif entre celles-ci. On peut faire à cet égard plusieurs remarques :

1) Nous avons supposé que les pseudo-potentiels du zinc et du cuivre étaient proportionnels à leur charge. Une telle approximation a été infirmée par l'étude de $\frac{c_{11}-c_{12}}{2}$. Ceci ne représente pas une critique fondamentale car la valeur d'une courbure du spectre de phonons en un point du spectre est très sensible aux détails des pseudo-potentiels. En revanche, cette remarque souligne la difficulté d'obtenir des résultats quantitatifs à partir de la notion de pseudo-potentiels.

2) L'emploi de pseudo-potentiels pour décrire la variation d'un spectre de phonons avec la température est assez discutable. En effet, même dans un système monoatomique, l'effet de la température est compliqué. Il se traduit d'abord par la variation du paramètre cristallin : ceci change les forces entre les ions de façon non triviale car la densité électronique moyenne intervient dans l'expression du potentiel self-consistant. Mais, du fait de l'agitation thermique, c'est une moyenne des forces entre les ions qu'il nous faut considérer, et cet effet peut être important. Afin de n'étudier que l'influence de la variation de l'ordre sur les spectres de phonons et d'éviter cette complication théorique, l'utilisation d'échantillons trempés à des températures différentes mais étudiés à une même température est sans doute souhaitable.

3) Finalement, l'emploi même de pseudo-potentiels scalaires dans les alliages ordonnés ne peut être justifié par la théorie des métaux. En effet, ceux-ci ne paraissent avoir de sens que dans les métaux normaux. En revanche, dans tous les alliages ordonnés dont l'ordre varie de façon notable avec la température, un des constituants au moins est un métal soit noble, soit de transition. Il ne faut donc considérer leur usage que comme une approximation plausible.

Il apparaît donc qu'une étude quantitative de l'influence de l'ordre sur les diverses propriétés physiques que nous avons indiquées se heurte encore à de nombreuses difficultés.

\section{BIBLIOGRAPHIE}

[1] Muto (T.), Sci. Pap. Inst. Phys. Chem. Res. Tokyo, 1936, 30, 99 .

[2] Krivograz (M. A.) et Matsyna (Z. A.), Sov. Phys. JETP, 1955, 1, 103.

[3] Gibson (J. B.), J. Phys. Chem. Solids, 1958, 4, 177.

[4] BEAL (M. T.), J. Phys. Chem. Solids, 1960, 15, 72 ; 1961, 18, 156

[5] PICK (R.), J. Phys. Chem. Solids, 1963, 24, 741.

[6] Beal (M. T.), J. Phys. Chem. Solids, 1961, 18, 150.

[7] Amroldi (G.), Adsente (M.) et Rimini (E.), Phys. Kond. Mat., 1964, 2, 180 ; Phil. Mag., 1964, 10, 43.

[8] Bragg (W. L.) et Wirliams (E. J.), Proc. Roy. Soc., 1934, A 145, 699; 1935, A 151, 540 .
[9] Gilat (G.) et Dolling (G.), Phys. Rev., 1965, 138, A 1053.

[10] Dev (B. N.) et Sen Gupta (S. P.), Indian J. Phys., 1964, 38, 341.

[11] Rodbeil (S.), Jacobs (I. S.) et al., Phys. Rev. Letters, 1963, 11, 10.

[12] Roussel, Thèse de $3^{\mathrm{e}}$ cycle, Paris, 1962, non publiée.

[13] Owen (E. A.) et Pickup (L.), Proc. Roy. Soc., 1934, A 145, 258.

[14] McManus (G. M.), Phys. Rev., 1963, 129, 2004.

[15] Shmatov (V. T.), Fiz. Metal. Metalloved., 1961, 11, $170 ; 1963,15,36$. 